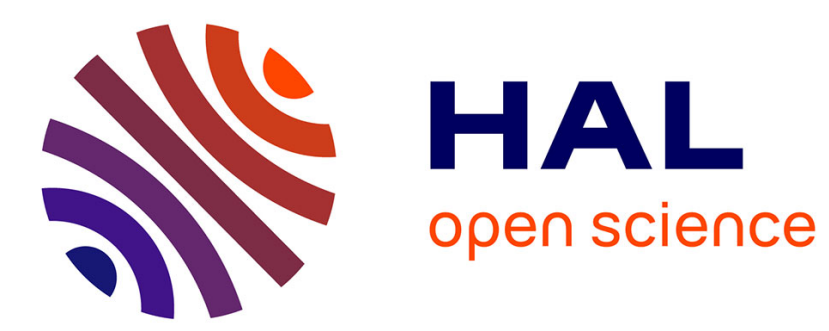

\title{
Analyse de dépôts PVD de tantale par diffraction X
}

\author{
L. Chekour, J. Krier, R. Halimi, A. Cornet
}

\section{To cite this version:}

L. Chekour, J. Krier, R. Halimi, A. Cornet. Analyse de dépôts PVD de tantale par diffraction X. Journal de Physique IV Proceedings, 1996, 06 (C4), pp.C4-251-C4-258. 10.1051/jp4:1996423 . jpa00254306

\section{HAL Id: jpa-00254306 https://hal.science/jpa-00254306}

Submitted on 1 Jan 1996

HAL is a multi-disciplinary open access archive for the deposit and dissemination of scientific research documents, whether they are published or not. The documents may come from teaching and research institutions in France or abroad, or from public or private research centers.
L'archive ouverte pluridisciplinaire HAL, est destinée au dépôt et à la diffusion de documents scientifiques de niveau recherche, publiés ou non, émanant des établissements d'enseignement et de recherche français ou étrangers, des laboratoires publics ou privés. 


\title{
Analyse de dépôts PVD de tantale par diffraction $\mathbf{X}$
}

\author{
L. Chekour, J. Krier*, R. Halimi et A. Cornet* \\ Unité de Recherche de Physique des Matériaux et Applications, Université de Constantine \\ * Laboratoire de Métallurgie, Corrosion et Matériaux, ENSAIS, Strasbourg, France
}

Résumé: Cette étude porte sur la détermination des contraintes résiduelles dans des films minces de tantale déposés par un procédé PVD (bombardement électronique et condensation sous vide) sur un substrat en acier. Les échantillons ont subi des recuits conventionnels (de 600 à $1100^{\circ} \mathrm{C}$ ) pour activer la diffusion du carbone, et former des carbures de tantale dans le dépôt. Ceci a pour effet, d'améliorer les propriétés mécaniques du revêtement. L'une de ces propriétés est caractérisée par le niveau de contraintes résiduelles. Pour les échantillons étudiés, ce sont des contraintes de compressionet leur valeur passe de $500 \mathrm{MPa}$, pour un échantillon non recuit, à $1500 \mathrm{MPa}$ pour un échantillon recuit à $1100^{\circ} \mathrm{C}$ durant une heure.

\begin{abstract}
The aim of this study is to determine residual stresses in Ta thin PVD deposits on steel. The samples are then annealed between 600 and $1100^{\circ} \mathrm{C}$ producing Ta carbides in the layer. These carbides increase the mechanical properties of the surface. They increase the compressive residual stresses in the Ta layer. Compressive stresses about $500 \mathrm{MPa}$ are measured without annealing and about $1500 \mathrm{MPa}$ for a sample annealed 1 hour at $1100^{\circ} \mathrm{C}$.
\end{abstract}

\section{1 - INTRODUCTION}

Pour améliorer les propriétés des surfaces, lors de sollicitations (thermiques, mécaniques, ...), les pièces sont souvent revêtues de dépôts métalliques ou autres, sous forme de couches ou de multicouches minces. Les matériaux conventionnels présentent généralement des propriétés non satisfaisantes, face aux utilisations de plus en plus exigeantes de l'industrie. Ces derniers, ont donc laissé place à de matériaux spéciaux de type céramique ou réfractaire, entre autres, qui ne peuvent être utilisés à l'état massif pour des raisons diverses; telles que leurs propriétés ou caractéristiques physiques insuffisantes (fragilité, usinage délicat,...) et leur coût de revient élevé. Pour remédier à ces problèmes, la solution a consisté à utiliser des matériaux classiques et d'en recouvrir la surface de matériaux présentant les propriétés requises.

L'utilisation de pièces revêtues connaît un développement important à cause de leurs propriétés électriques, optiques, tribologiques et/ou leur résistance à la corrosion. Plusieurs types de matériaux sont ainsi déposés sur des substrats par divers procédés ( $P V D, C V D, \ldots)[1-3]$. 
Le revêtement obtenu par déposition (PVD) du tantale sur un substrat d'acier (XC100), auquel on fait subir des recuits conventionnels, fait l'objet de cette étude. L'activation de la diffusion, par la température de recuit, du carbone du substrat vers la couche de tantale entraîne la formation de carbures qui confèrent au dépôt de bonnes caractéristiques mécaniques. Ces propriétés peuvent cependant être influencées par la présence de contraintes résiduelles. Le but de ce travail est d'évaluer le niveau de ces contraintes dans le revêtement de tantale.

\section{PREPARATION DES ECHANTILLONS}

Les dépôts de tantale sur un acier XC100 (tableau 1) ont été réalisées sous vide $\left(10^{-6}\right.$ Torr) par évaporation d'une cible de tantale sous bombardement électronique. Pour augmenter l'adhésion et la densité du revêtement le substrat a été porté à une température de $450{ }^{\circ} \mathrm{C}$ en utilisant un rayonnement photonique pendant toute l'opération de dépôt. L'épaisseur finale de la couche de tantale est d'environ $4 \mu \mathrm{m}$. Après l'opération, les échantillons sont concervés dans l'enceinte sous la même pression jusqu'à refroidissement.

Pour améliorer les propriétés de la couche de tantale, des recuits conventionnels ont été effectués dans un tube de quartz sous vide $\left(2.10^{-5}\right.$ Torr) à des températures variant de 600 à $1100^{\circ} \mathrm{C}$.

Tableau 1: Composition chimique du substrat XC100 [4].

Chemical composition of the substract.

\begin{tabular}{|c|c|c|c|c|c|c|c|}
\hline $\begin{array}{c}\text { Nuance } \\
\mathrm{XC} 100\end{array}$ & $\mathrm{C}$ & $\mathrm{Mn}$ & $\mathrm{S}$ et P & $\mathrm{Si}$ & $\mathrm{Cr}$ & $\mathrm{Ni}$ & $\mathrm{Cu}$ \\
\hline $\min$. & 0.95 & 0.25 & $\mathrm{P}<0.030$ & 0.15 & 0.15 & 0.20 & 0.20 \\
\hline $\max$. & 1.05 & 0.45 & $\mathrm{~S}<0.025$ & 0.30 & - & - & - \\
\hline
\end{tabular}

( température de trempe à $1^{\prime}$ huile $: 800^{\circ} \mathrm{C}$, dureté : $61 \mathrm{HRC}$ )

\section{RESULTATS}

\subsection{Microstructure}

L'observation au microscope optique du substrat non recuit révèle une microstructure (figure 1a) à matrice ferritique dans laquelle des globules fins de cémentite (gris) sont répartis au hasard et alignés par endroits. Les échantillons recuits possèdent une structure lamellaire composée de bandes alternées de ferrite et de cémentite (figure 1b). Les substrats recuits ou non, ne présentent pas de structure à orientations préférentielles.

La morphologie superficielle des couches de tantale (structure cubique centrée) a un aspect colonnaire (figure 2). Les bandes parallèles sont tapissées de particules de densité croissante avec la température de recuit. Ces bandes sont aussi interrompues par des micro-fissures transversales (figure 2a). L'augmentation de la température de recuit active la diffusion du carbone du substrat vers le dépôt. Cette diffusion, donne naissance à des carbures qui vont, au fur et à mesure de leur formation combler, les micro-fissures (figure $2 \mathrm{~b}$ ). On citera le Ta2C, la phase $\xi$ et le TaC. Ce dernier carbure est thermodynamiquement le plus stable. A partir de $1000{ }^{\circ} \mathrm{C}$, les carbures Ta2C et la phase $\xi$ disparaissent pour laisser place au carbure TaC [7]. 

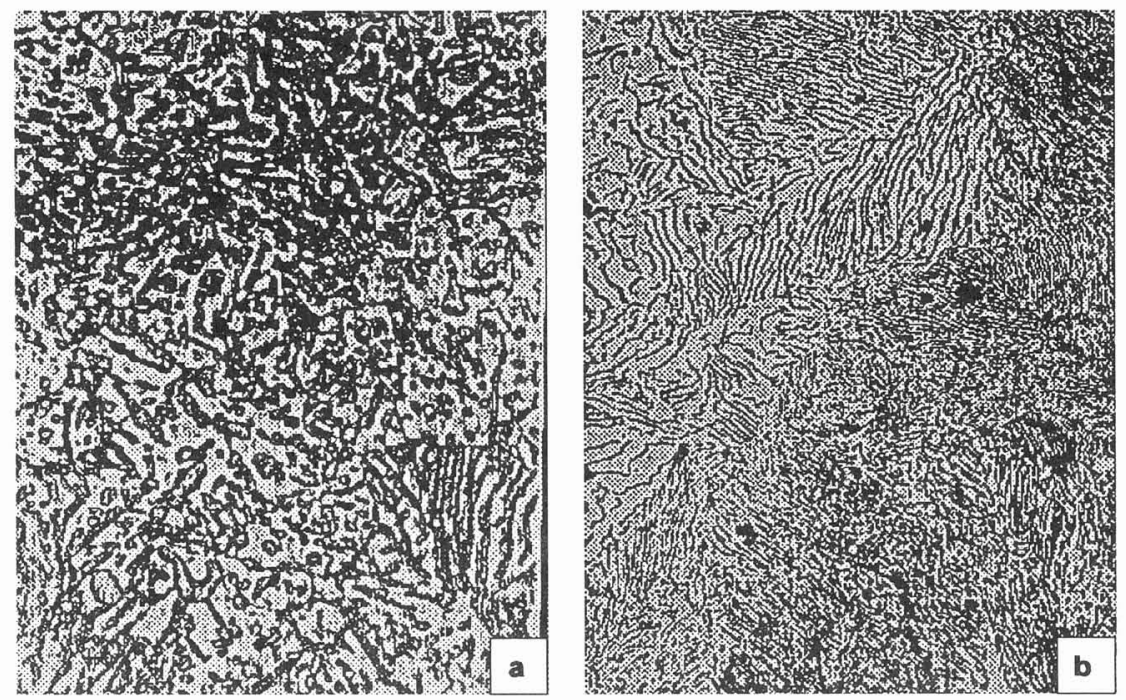

Figure 1: Microstructure du substrat $\mathrm{XC100,} \mathrm{a)} \mathrm{avant} \mathrm{recuit,} \mathrm{b)} \mathrm{après} \mathrm{recuit} 1100^{\circ} \mathrm{C}$ pendant 1 heure

XC100 microstructure, a) before annealing, b) after annealing 1 hour at $1100^{\circ} \mathrm{C}$.
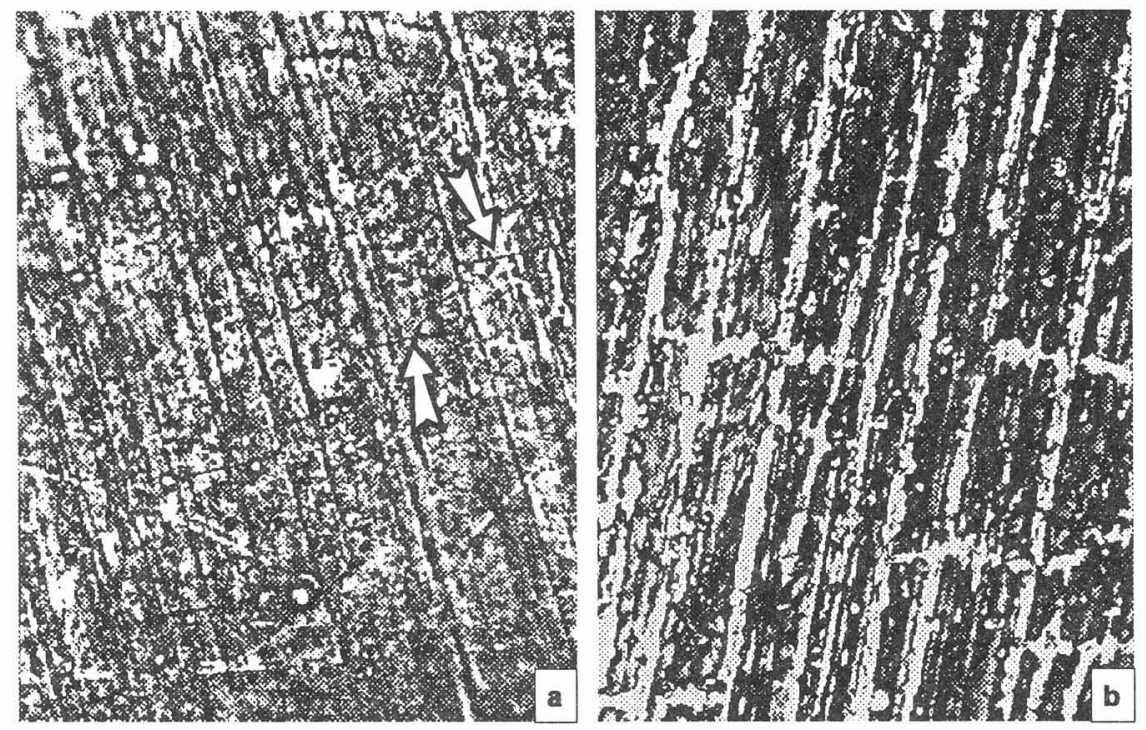

Figure 2: Microstructure du revêtement, a) échantillon non recuit, b) échantillon recuit $1100^{\circ} \mathrm{C}$ pendant 1 heure.

Deposit's microstructure, a) not annealed, b) annealed 1 hour at $1100^{\circ} \mathrm{C}$. 


\subsection{Les phases en présences}

L'analyse par diffraction $\mathrm{X}$ permet de déterminer les phases présentes dans le dépôt et leur évolution en fonction de la température de recuit. Ainsi, le dépôt se compose, avant recuit, uniquement de tantale (figure $3 a$ ). La figure $3 b$ montre qu'après un recuit d'une heure à $1100^{\circ} \mathrm{c}$ tout le tantale s'est transformé en carbure de tantale, $\mathrm{TaC}$.

a

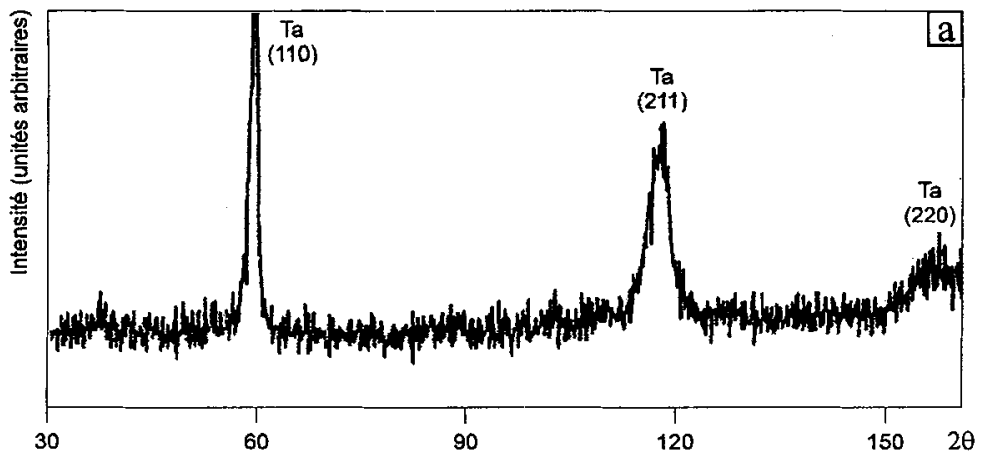

b

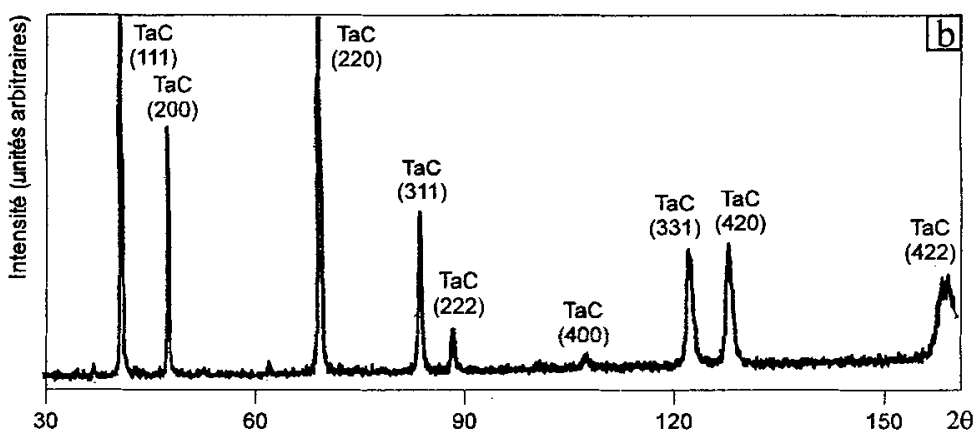

Figure 3: Diffractogrammes du revêtement de $\mathrm{Ta}$, a) échantillon non recuit (tube à anticathode de $\mathrm{Cr}$ ) et b) échantillon recuit à $1100^{\circ} \mathrm{C}$ pendant 1 heure (tube à anticathode de $\mathrm{Co}$ ) Diffractogram of the Ta deposit, a) not annealed (Cr X-ray tube), b) annealed 1 hour at $1100^{\circ} \mathrm{C}$ (Co X-ray tube).

\subsection{Les contraintes résiduelles}

La détermination des contraintes à partir de la loi des « $\sin ^{2} \psi$ [5] s'effectue à partir de la mesure des distances interréticulaires pour 9 angles $\psi$, dans 2 directions $\varphi$. Ces angles définissent la direction de mesure. Pour les aciers, on utilise généralement le déplacement du doublet de la raie $\{211\}$ qui se situe à $2 \theta=156^{\circ}$ dans le cas de l'utilsation d'un tube à anticathode de Chrome. Les diffractogrammes de la figure 3 permettent de choisir la raie $\{211\}$ du tantale pour des températures de recuit inférieures à $700^{\circ}$ et la raie $\{311\} \mathrm{du} \mathrm{TaC}$ pour des température de recuit supérieures. Des mesures seront également effectuées sur la raie $\{420\}$ du TaC dans le cas de l'utilsation d'un tube à anticathode de cobalt. 
Les constantes élastiques radiocristallographiques (CER) de ces différentes raies sont calculées à partir des constantes élastiques de monocristaux de fer, tantale et carbure de tantale données dans le tableau 2.

Les valeurs de ces constantes permettent de calculer le coefficient d'anisotropie élastique $\mathrm{A}=$ $\left(\mathrm{S}_{11}-\mathrm{S}_{12}\right) \mathrm{S}_{44}$, du tantale et du carbure de tantale $(\mathrm{TaC})$. Les résultats obtenus montrent que ces matériaux ont un comportement élastique pratiquement isotrope $\left(\mathrm{A}_{\mathrm{Ta}}=0.98 ; \mathrm{A}_{\mathrm{TaC}}=\right.$ 0.99), lisotropie correspondant à $\mathrm{A}=1$.

Tableau 2: Constantes élastiques $\left(\times 10^{6} \mathrm{MPa}^{-1}\right)$ [6] Elastic Constants $\left(x 10^{6} M \mathrm{~Pa}^{-1}\right)$.

\begin{tabular}{|c|c|c|c|c|c|c|c|}
\cline { 2 - 8 } \multicolumn{1}{c|}{} & $\mathrm{E}(\mathrm{GPa})$ & $v$ & $\mathrm{~S}_{11}$ & $\mathrm{~S}_{44}$ & $\mathrm{~S}_{12}$ & $1 / 2 \mathrm{~S}_{2}(\mathrm{hkl})$ & $\mathrm{S}_{1}(\mathrm{hkl})$ \\
\hline$(211) \mathrm{Ta}$ & 189 & 0.34 & 5.3 & 14.5 & -1.80 & 7.21 & -1.84 \\
\hline$(311)(420) \mathrm{TaC}$ & 303 & 0.27 & 3.29 & 8.35 & -0.88 & 4.17 & -0.88 \\
\hline$(211) \mathrm{Fe}_{\alpha}$ & 210 & 0.30 & 4.80 & 12.3 & 1.43 & 6.22 & -1.43 \\
\hline
\end{tabular}

Les contraintes, déterminées à partir des courbes de la figure $4 \mathrm{a}$, sont regroupés dans le tableau 3 . On observe que les contraintes sont négligeables dans le substrat avant déposition. Les contraintes dans le revêtement sont de l'ordre de $500 \mathrm{MPa}$ avant recuit et passent par un maximum vers $1000^{\circ} \mathrm{C}$ pour un recuit d'une heure. On remarque également pour l'échantillon 6 que la contrainte augmente avec la durée du recuit.

La faible profondeur de pénétration des rayons $X$ dans le tantale $(\tau(\mathrm{Cr})=0,56 \mu \mathrm{m}, \tau(\mathrm{Co})=$ $0,12 \mu \mathrm{m}$, valeurs calculées pour $\psi=0$ ) et les carbures de tantale, écarte la contribution du substrat à la diffraction lors de la mesure sur le revêtement.

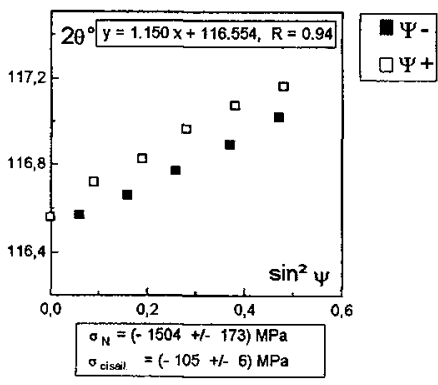

a)

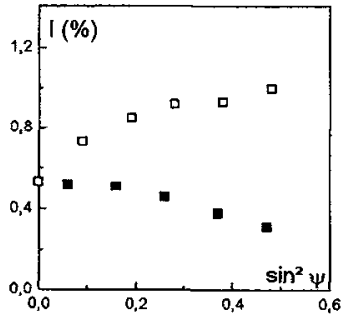

b)

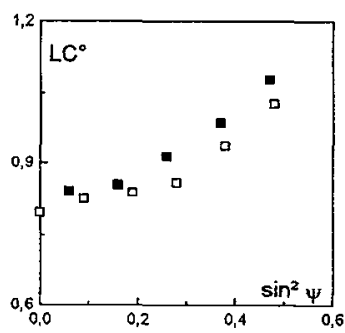

c)

Figure 4: Echantillon 6, recuit à $1100^{\circ} \mathrm{C}$, pendant 1 heure (Tube à anticathode de $\mathrm{Cr}$ ). Variation des caractéristiques de la raie (311)TaC en fonction de $\sin ^{2} \Psi:$ a) position $2 \theta$; b) intensité ; c) largeur de corde

Sample 6, annealed 1 hour at $1100^{\circ} \mathrm{C}\left(\mathrm{Cr} X\right.$-ray tube), a) $\left.2 \theta v s . \sin ^{2} \Psi, b\right)$ intensity vs. $\sin ^{2} \Psi$, c) breadth width vs. $\sin ^{2} \Psi$. 
Les mesures effectuées avec le rayonnement de $\mathrm{Cr} \mathrm{K} \alpha$ et le rayonnement de $\mathrm{Co} \mathrm{K} \alpha$ sont identiques en terme de contrainte et de largeur à mi-hauteur. Ceci permet de dire qu'il n'y a pas de gradient de contraintes en profondeur.

La figure $4 \mathrm{~b}$, représentant la variation de lintensité en fonction de $\sin ^{2} \psi$, montre la présence d'une texture cristallographique qu'il faudrait intégrer pour une meilleure détermination des contraintes. La figure 3c représente la variation de la largeur de la corde à mi-hauteur qui caractérise les microdéformations induites par les distorsions fortes du réseau de tantale.

Tableau 3: Valeurs des contraintes résiduelles dans le substrat brut et dans le revêtement de tantale.

Residual stresses in the substrate and in the Ta deposit.

\begin{tabular}{|c|c|c|c|c|c|}
\hline Echantillons & plans (hkl) & $\mathrm{T}^{\circ} \mathrm{C}$ (Recuit) & $\begin{array}{c}\text { Temps- } \\
\text { maintien }\end{array}$ & $\sigma_{\mathrm{n}}$ (MPa) & $\sigma_{\mathrm{t}}$ (MPa) \\
\hline $\mathrm{XC100}$ & $(211)$ & - & - & $-26+/-3$ & $-6+/-1$ \\
\hline 1 & $(211)$ & - & - & $-532+/-46$ & $+16+/-22$ \\
\hline 2 & $(211)$ & 600 & $1 \mathrm{~h}$ & $-950+/-20$ & $-98+/-4$ \\
\hline 3 & $(311)$ & 900 & $1 \mathrm{~h}$ & $-1498+/-39$ & $-46+/-7$ \\
\hline 4 & $(222)$ & 900 & $3 \mathrm{~h} 20$ & $-1776+/-67$ & $-28+/-14$ \\
\hline 5 & $(311)$ & 1000 & $1 \mathrm{~h}$ & $-1639+/-56$ & $-19+/-12$ \\
\hline 6 & $(311)$ & 1100 & $1 \mathrm{~h}$ & $-1504+/-173$ & $-105+/-6$ \\
\hline
\end{tabular}

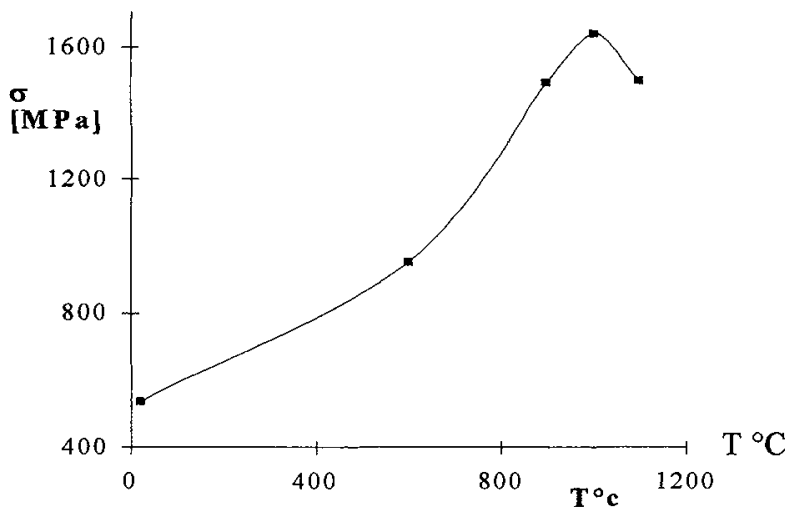

Figure 5: Evolution de la contrainte avec la température de recuit (maintien 1 heure) Stress vs. 1 hour annealing temperature.

\section{DISCUSSION}

Dans pratiquement tous les types de revêtements des contraintes résiduelles apparaissent [810]. Le niveau de ces contraintes dépend du procédé de déposition utilisé, des paramètres liés au procédé lui même et aux traitements subis par le matériau revêtu. Les types de substrats et matériaux déposés ont aussi une incidence sur les contraintes. 
Les revêtements obtenus par pulvérisation cathodique présentent généralement des contraintes de compression de très forte intensité [8-10]. Dans le cas du revêtement de tantale, la déposition PVD s'est effectuée par bombardement électronique à l'aide d'un canon à électrons. L'avantage de cette méthode est l'obtention de films propres, car la contamination est minimisée par l'absence de gaz rare dans l'enceinte. Le faible taux d'impuretés implique naturellement moindre distorsion du réseau, et par la même, un niveau de contrainte relativement bas par rapport aux revêtements obtenus par d'autres procédés utilisant un gaz réactif dans l'enceinte. En effet, au niveau de l'échantillon brut (non recuit), la contrainte trouvée est d'environ $550 \mathrm{MPa}$, alors que dans des revêtements de molybdène, par exemple, les contraintes normales sont supérieures à $1000 \mathrm{MPa}$ [9] et ce, quelle que soit la tension de polarisation du substrat.

La contrainte globale existant dans les différents types de revêtements peut être d'origine thermique ou intrinsèque. La contrainte d'origine thermique est due à la différence des coefficients de dilatation thermique du substrat et du dépôt. La seconde origine des contraintes est, par contre, due à l'existence de défauts de germination et de croissance (lacunes, interstitiels, dislocations, impuretés,...).

Tableau 4: Caractéristiques mécaniques du fer $\alpha$, du tantale et du carbure de tantale (TaC) Elastic caracteristics of $\alpha$ iron, Ta and TaC.

\begin{tabular}{|c|c|c|c|c|}
\cline { 2 - 5 } \multicolumn{1}{c|}{} & $\begin{array}{c}\mathrm{E} \\
(\mathrm{GPa})\end{array}$ & $\begin{array}{c}\mathrm{HV} \\
\left(\mathrm{kg} / \mathrm{mm}^{2}\right)\end{array}$ & $\begin{array}{c}\text { structure } \\
\text { cristalline }\end{array}$ & $\begin{array}{c}\text { coef. dilatation thermique. } \alpha \\
\left(10^{-6} /{ }^{\circ} \mathrm{C}\right)\end{array}$ \\
\hline $\mathrm{Fe} \alpha$ & 210 & 400 & $\mathrm{CC}$ & 12 \\
\hline $\mathrm{Ta}$ & 189 & 500 & $\mathrm{CC}$ & 6.5 \\
\hline $\mathrm{TaC}$ & 303 & 2400 & $\mathrm{CFC}$ & - \\
\hline
\end{tabular}

Dans le cas des revêtements de tantale, les contraintes résiduelles trouvées (500 à $1700 \mathrm{MPa}$ ) augmentent avec la température du recuit (figure 6) et le temps de maintien. La diffusion du carbone dans le tantale, activée par la température des recuits et facilitée par le faible rayon atomique du carbone relativement à celui du tantale $\left.\left(\mathrm{R}_{(\mathrm{Ta}}{ }^{+3}\right)=0.72 \mathrm{~A}, \mathrm{R}\left(\mathrm{C}^{+4}\right)=0.16 \mathrm{~A}\right)$, initie la formation de carbures de différents types ( $\mathrm{TaC}, \mathrm{Ta} 2 \mathrm{C}$, phase $\xi$ ) [7]. Ces carbures présentent un module d'Young, un coefficient de dilatation thermique et un niveau de dureté plus élevé (tableau 4) que ceux du substrat. L'écart s'amplifie avec l'augmentation de la température du recuit.

La dilatation, plus importante, du substrat par rapport au dépôt, au moment de la déposition du tantale $\left(450^{\circ} \mathrm{C}\right)$, et surtout au cours des recuits, peut expliquer le signe des contraintes qui apparaissent au cours du refroidissement. Une partie des contraintes résiduelles est donc, d'origine thermique. Cependant, la source intrinsèque des contraintes n'est pas à négliger. en effet, malgré l'absence de gaz rare dans l'enceinte de préparation des échantillons, les spectres des diffraction X et SIMS [7] montrent la présence d'oxydes et de nitrures de tantale. D'autre part, l'insertion des atomes de carbone dans le réseau de tantale par diffusion, durcit le revêtement, et provoque des distorsions du réseau qui donnent naissance à des contraintes de compression. La contrainte mesurée sur l'échantillon non recuit est relativement faible (530 MPa). Cela est dû, probablement, à la présence de micro-fissures provoquées par la relaxation des contraintes d'origine thermique, pendant le refroidissement de l'échantillon. La formation croissante de carbures pendant les différents recuits de diffusion, dans les autres échantillons, 
tend à combler les micro-fissures et donner naissance à des contraintes de compression qui atteignent $1700 \mathrm{MPa}$. La micro-dureté varie dans le même sens pour atteindre une valeur maximale de $2400 \mathrm{~kg} / \mathrm{mm}^{2}$. Le recouvrement des micro-fissures par les carbures de tantale se généralise presque totalement pour les échantillons recuits à $1100^{\circ} \mathrm{C}$.

\section{CONCLUSIONS}

Ce travail a porté sur l'analyse des contraintes résiduelles par diffraction des rayons $\mathrm{X}$, dans des revêtements de tantale (épaisseur $4 \mu \mathrm{m}$ ) obtenus par évaporation de tantale pur $(99.99 \%)$ sous bombardement électronique.

Le recours à des recuits conventionnels, permettant la diffusion du carbone dans la couche de tantale, a permis l'obtention d'un complexe substrat-revêtement, caractérisé par une bonne cohésion et une bonne adhésion au niveau de l'interface. La nature compressive et le niveau des contraintes trouvées $\left(\sigma_{\max } \cong 1700 \mathrm{MPa}\right.$ ) confèrent au revêtement de bonnes propriétés d'utilisation. Un choix judicieux de la température de recuit et du temps de maintien des échantillons doit conduire à des caratéristiques optimales du revêtement.

\section{Références:}

[1] Handbook of Thin film Technology, LI. Maissen and R. Gland. Eds, Mc Graw Hill Book . Company, New York (1970).

[2] Manuel de traitements de surfaces à l'usage des bureaux d'étude, CETIM, Paris (1987).

[3] R.F Bunshah, Deposition Technologies for Films and Coatings Developpements and Applications, Noyes Publications, Park Ridge, New Jersey (1982).

[4] Recueil de normes des produits sidérurgiques (nuances et qualité), Tome1, AFNOR, Paris (1976) p.264.

[5] L. Castex, J.L. Lebrun, G. Maeder et J.M. Sprauel, "Détermination des contraintes résiduelles par diffraction des rayons $X^{\prime \prime}$. Publication $n^{\circ}$ 22, ENSAM, Paris (1981).

[6] Theory of dislocations, J.P. Hirth and J. Lothe Eds, Wiley, New York (1982).

[7] Y. Hadjar, Thèse de Magister, Université de Constantine (1994).

[8] M. Zaouali, Thèse de Doctorat, ENSAM Paris (1990).

[9] P. Gergaud, Thèse de Doctorat, ENSAM Paris (1992). 